\title{
LIPOMA GIGANTE DE ESÔFAGO: RELATO DE TRATAMENTO CIRÚRGICO
}

\section{LARGE ESOPHAGEAL LIPOMA: REPORT OF SURGICAL TREATMENT}

Bruno Amantini Messias ${ }^{1}$, TCBC-SP; Karin Silva Ferreira ${ }^{2}$; Gabriel Belnuovo Vieira ${ }^{2}$; Marcos Dias

Nunes de Moraes ${ }^{1}$; Roberto Eduardo Donoso-Rivero ${ }^{1}$; Érica Rossi Mocchetti ${ }^{1}$.

\section{RESUMO}

Introdução: Lipoma esofágico é uma patologia extremamente rara e patologicamente benigna. Geralmente são assintomáticos e descobertos incidentalmente em exames de imagem. A principal localização é no terço superior do esôfago. Disfagia, massa regurgitada e sensação de desconforto na gargante são as principais queixas dos pacientes. O tratamento endoscópico é uma opção em lesões pequenas, entretanto, lesões maiores devem ser preferencialmente abordadas por cirurgia convencional. Relato do caso: Relatamos o caso de uma paciente jovem com história de disfagia e dor torácica ha 2 anos. Referia saída de lesão pela cavidade oral após episódio de tosse. Submetida a endoscopia digestiva que evidenciou lesão pediculada esofágica e tomografia computadorizada que visualizou lesão esofágica de 20 centimetros. Com base nesses achados, foi optado pelo tratamento cirúrgico. Conclusão: o lipoma esofágico é uma patologia rara e que geralmente necessita alto índice de suspeição, exame endoscópico e de imagem para o correto diagnóstico. O tratamento cirúrgico e endoscópico são as principais modalidades terapêuticas.

Palavras-chave: Lipoma. Neoplasias. Esôfago.

\section{ABSTRACT}

Introduction: Esophageal lipoma is an extremely rare and pathologically benign condition. They are usually asymptomatic and discovered incidentally on imaging studies. The main location is in the upper third of the esophagus. Dysphagia, regurgitated mass and feeling of discomfort in the throat are the main complaints of patients. Endoscopic treatment is an option in small lesions, however, larger lesions should preferably be addressed by conventional surgery. Case report: we report the case of a young patient with a 2-year history of dysphagia and chest pain. She reported that the lesion had left the oral cavity after an episode of coughing. Submitted a digestive endoscopy that evidenced pedicled esophageal lesion and computed tomography that visualized esophageal lesion measuring 20 centimeters. Based on these findings, surgical treatment was chosen. Conclusion: Esophageal lipoma is a rare pathology and usually requires a high level of suspicion, endoscopic and imaging exams for a correct diagnosis. Surgical and endoscopic treatment are the main therapeutic modalities.

Keywords: Lipoma. Neoplasms. Esophagus.

\section{INTRODUÇÃO}

A doença diverticular do cólon é uma afecção comum que acomete metade Os tumores benignos do esôfago são muito raros, constituindo apenas $0,5-0,8 \%$ de todas as neoplasias esofágicas. Dentre as lesões benignas, os leiomiomas são as neoplasias mais incidentes, acometendo aproximadamente $60 \%$ dos pacientes.

Os lipomas são responsáveis por apenas $1 \%$ dos casos, enquanto os cistos e pólipos esofágicos são encontrados em até $25 \%$ dos pacientes ${ }^{1,2}$. Os lipomas podem ser encontrados em todos os segmentos do trato gastrointestinal (TGI). As lesões no cólon são as mais comuns, seguidas, em ordem decrescente de aparecimento, por lesões no intestino delgado, no estômago e, por último, no esôfago ${ }^{1,2}$.

Lipoma no trato gastrointestinal é uma patologia mais prevalente no gênero feminino. Já na topografia esofágica, essas lesões, são mais prevalentes no gênero masculino, numa relação de 27:13. Os lipomas podem acometer pacientes de 4 a 80 anos de idade, entretanto, tem uma predileção maior por pacientes na $5^{\mathrm{a}}$ década de vida² .

A formação exata dos lipomas ainda é incerta, no entanto, uma teoria propõe uma forte associação entre trauma prévio e o surgimento do lipoma no local2.

\footnotetext{
${ }^{1}$ Hospital Geral de Carapicuíba, Departamento de Cirurgia - Carapicuíba - SP - Brasil

${ }^{2}$ Centro Universitário São Camilo, Curso de medicina - São Paulo - SP - Brasil
} 
A maioria das lesões são assintomáticas devido ao pequeno tamanho, porém, lesões maiores do que $4 \mathrm{~cm}$ podem desencadear sintomas como disfagia, regurgitação ou dispneia ${ }^{2,3}$.

Relatamos um caso raro de lipoma esofágico gigante pediculado em uma paciente jovem que apresentava disfagia progressiva e foi submetida a tratamento cirúrgico por cervicotomia.

\section{RELATO DO CASO}

Paciente de 38 anos, gênero feminino, veio ao ambulatório com história de dor em região torácica há 2 anos associada a tosse, disfagia para sólidos e líquidos. Relatava que durante um episódio de tosse houve a saída de uma massa pela boca com retorno espontâneo. Paciente negava perda de peso, patologias prévias ou cirurgias. Ao exame físico não apresentava nenhuma anormalidade.

$\mathrm{Na}$ investigação inicial foi realizado endoscopia digestiva alta (EDA). À EDA foi identificada uma lesão pediculada no esôfago proximal, ocupando quase completamente a luz do órgão, com uma extensão aproximada de 20 centimetros. Não foi identificado lesões na mucosa ou sinais de ulceração. Devido ao grande tamanho da lesão, o tratamento endoscópico não foi exequível.

Para uma melhor avaliação diagnóstica e adequado planejamento terapêutico foi realizado estudo tomográfico do pescoço, tórax e abdome. No exame tomográfico foi visualizada extensa lesão endoluminal esofágica com aproximadamente 20 centímetros extensão que acarretava dilatação do órgão (Figuras 1, 2 e 3).

Com base no resultado desses exames e na impossibilidade do tratamento endoscópico, foi proposto à paciente a abordagem cirúrgica por via cervical. O procedimento foi programado de maneira eletiva após a realização de exames préoperatórios e avaliação préanestésica. A cirurgia foi realizado sob anestesia geral.

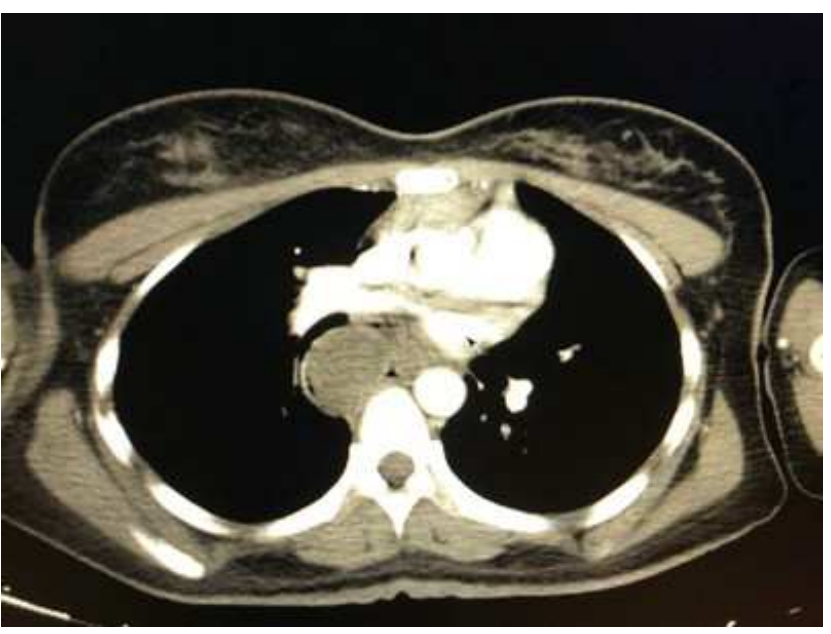

Figura 1: Imagem tomográfica em corte axial mostrando lesão esofágica ocupando quase a totalidade do órgão.

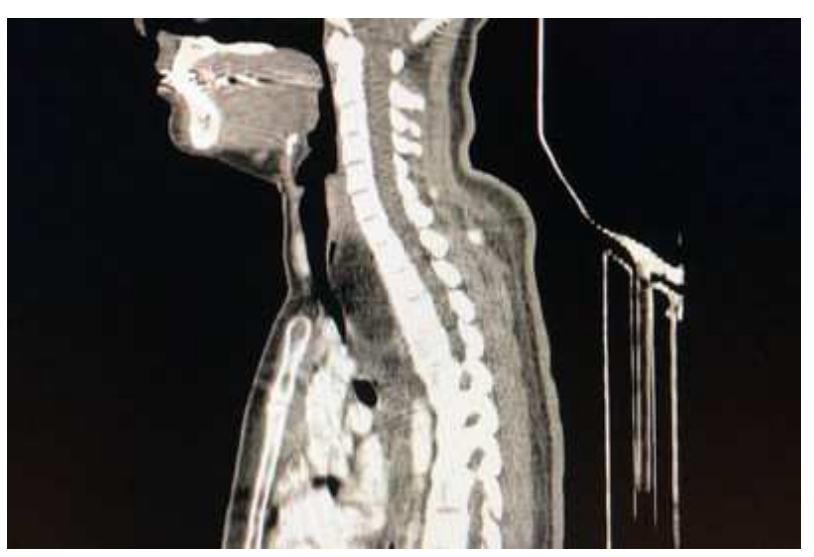

Figura 2: Imagem tomográfica em corte sagital evidenciando lesão endoluminal esofágica, que se estende até esôfago distal.

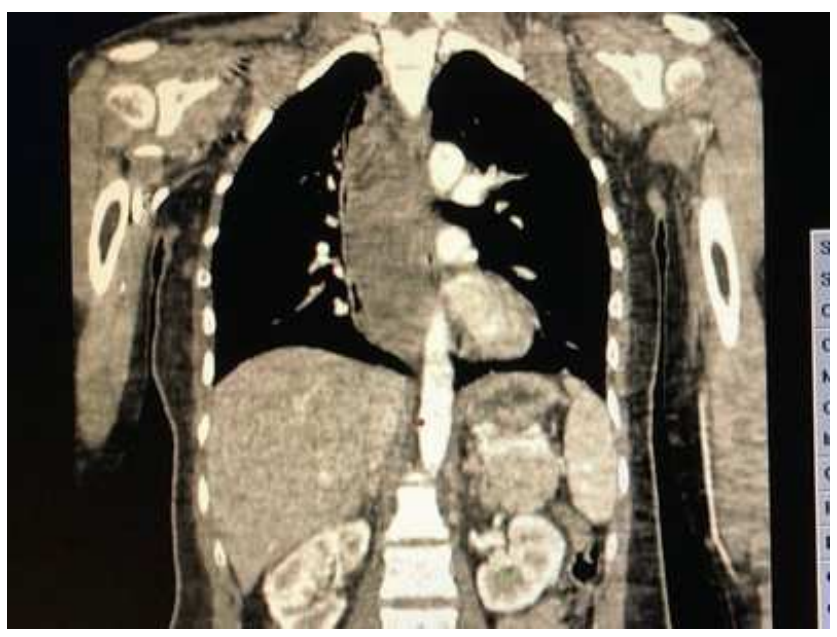

Figura 3: Imagem tomográfica em corte coronal mostrando dilatação esofágica às custas de grande lesão pedunculada esofágica. 
O procedimento iniciou-se com uma cervicotomia transversa à esquerda e dissecção por planos. Foi dissecado e isolado a cartilagem tireoidiana com posterior manobra de Tapia para melhor visualização da hipofaringe, com preservação do nervo laringeo recorrente. Optado por faringo esofagotomia lateral esquerda com extensão de 6 centímetros.

Após abertura do esôfago foi luxado lesão pediculada esofágica de aproximadamente 25 centímetros de extensão (Figura 4).

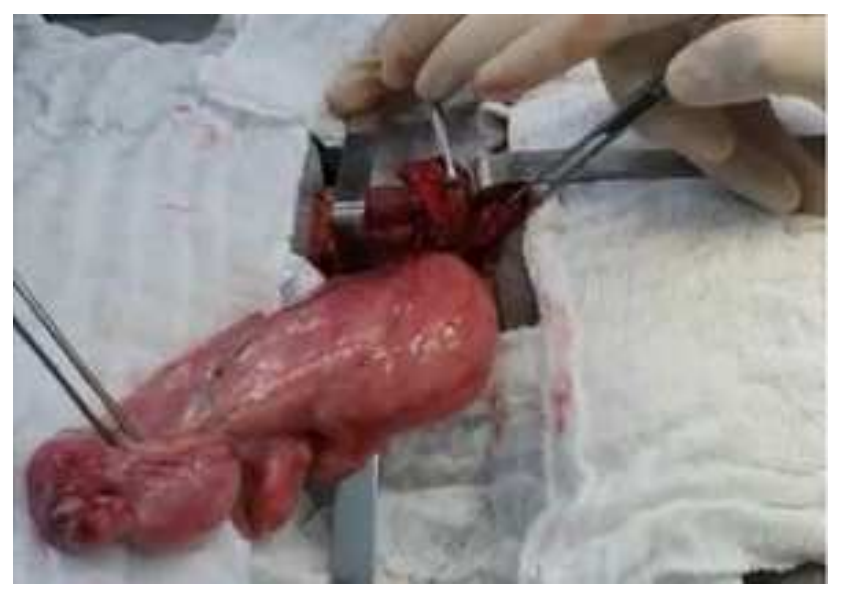

Figura 4: Imagem do intra-operatório após faringoesofagotomia e luxação da lesão pedunculada esofágica com extensão de aproximadamente 20 centimetros.

Devido ao pedículo pequeno da lesão, foi realizado ressecção transmural de aproximadamente $3 \mathrm{~cm}$ de extensão no esôfago e $2 \mathrm{~cm}$ na faringe, com posterior fechamento da faringo esofagotomia com fio de Vicryl 3.0 em pontos separados e confecção de retalho da musculatura prétireoidiana para reforço da síntese. Após a sintese foi optado pela drenagem cervical com dreno de Penrose. Realizado passagem de sonda nasoenteral para início precoce da alimentação enteral. Procedimento transcorreu sem intercorrências e a paciente foi encaminhada a UTI para suporte intensivo.

Recebeu alta da UTI no $10^{\circ}$ pósoperatório (PO). Iniciado dieta enteral em menos de 24 horas após o procedimento. A alta hospitalar aconteceu no $5^{\circ}$ dia de pósoperatório. Duas semanas após o procedimento, foi realizado deglutograma de controle, não sendo identificado nenhuma anormalidade. $\mathrm{O}$ anatomopatológico da lesão teve como diagnóstico lipoma e o perfil imunohistoquímico também foi condizente com o diagnóstico anterior. Paciente segue em acompanhamento ambulatorial sem intercorrências no período.

\section{DISCUSSÃO}

Tumores esofágicos benignos são incomuns, representando menos de $1 \%$ de todas as neoplasias esofágicas. Os lipomas esofágicos são ainda mais raros, sendo responsáveis por apenas $0,4 \%$ dos tumores gastrointestinais. Outros tumores que podem ser encontrados no esôfago são: leiomiomas, pólipos, papilomas, neurofibromas, além de tumores malignos. Os tumores estromais gastrointestinais, leiomiossarcoma maligno e carcinoma escamoso são importantes diagnósticos diferenciais, pois requerem um tratamento mais agressivo 2,3 .

A maioria dos lipomas são lesões assintomáticas e achadas incidentalmente em exames de imagem. Os sintomas estão muito relacionados com o seu tamanho. As lesões lipomatosas no TGI com menos de $1 \mathrm{~cm}$ geralmente não cursam com sintomatologia, entretanto, lipomas com mais de $4 \mathrm{~cm}$ são sintomáticos em mais de $75 \%$ dos pacientes. Cerca de $85 \%$ dos lipomas no esôfago são clinicamente assintomáticos ${ }^{2}$. Os pacientes sintomáticos relatam disfagia, regurgitação, melena recorrente e/ou epigastralgia ${ }^{2,4}$. A queixa mais frequente na maioria dos casos é a $\operatorname{disfagia}^{1,2}$.

A localização mais comum do pedículo tumoral é no esfincter esofágico superior. Embora o lipoma seja patologicamente benigno, pode apresentar alto potencial de complicações clínicas devido a sua localização. Lipomas gigantes, geralmente no terço superior do esôfago, podem causar asfixia por obstrução das vias aéreas, secundaria a compressão mecânica direta da laringe ${ }^{3-5}$. Outra complicação, menos comum, é a degeneração maligna de sua extremidade distal, possivelmente atribuída ao efeito crônico de secreções gástricas ${ }^{3}$.

Estabelecer o diagnóstico de um lipoma esofágico requer uma história clínica detalhada, exames de imagem e exame endoscópico minucioso, além de alta suspeição clínica. Com base nessas características, podemos lançar mão de vários métodos de imagem para auxiliar no diagnóstico. A tomografia computadorizada, ultrassonografia endoscópica (USGE), 
radiografia e ressonância magnética estão entre os principais métodos diagnósticos. No exame radiográfico, os lipomas se apresentam como falhas de enchimento, enquanto que, na tomografia o achado de uma lesão homogênea com densidade de gordura é muito sugestivo de lipoma. Já os leiomiomas, que são tumores intramurais, se apresentam como lesão com densidade de partes moles.

A ressonância magnética apresenta um hipersinal em T1, que se torna hipointenso em imagens com supressão de gordura. A ultrassonografia endoscópica (USGE) demonstrou um importante rendimento diagnóstico na avaliação dos tumores submucosos esofágicos ${ }^{3}$. No USGE, os lipomas aparecem como lesões hiperecóicas e homogêneas com margens externas lisas, surgindo na camada submucosa, enquanto que os lipossarcomas podem se apresentar como uma imagem heterogênea com áreas de ecogenicidade aumentada $^{2,4}$. Na endoscopia, os lipomas esofágicos apresentam-se como uma lesão de superficie lisa, amarelada e com fácil retração da mucosa quando pressionada. Em contraste, as neoplasias malignas são duras, irregulares e com mucosa friável e ulcerada ${ }^{2,4}$. Apesar dos vários métodos de imagem utilizados, apenas o exame anatomopatológico define o diagnóstico de lipoma.

O tratamento dos lipomas esofágicos pode ser cirúrgico ou endoscópico. A ressecção cirúrgica é recomendada se o tratamento endoscópico falhar ou a lesão for maior que $4 \mathrm{~cm}$. O tratamento endoscópico de lipomas esofágicos gigantes é tecnicamente dificil devido a sua vascularização e o risco de perfuração do esôfago. Apenas alguns relatos de casos foram publicados com essa técnica ${ }^{4}$.

Apesar da abordagem endoscópica e cirúrgica terem sido descritas com bons resultados, a escolha da intervenção deve ser considerada cuidadosamente. O risco de colapso das vias aéreas durante a execução do procedimento, o tamanho do tumor, o local do implante e o conteúdo vascular de seu pedículo devem nortear a escolha da melhor via de tratamento. A abordagem minimamente invasiva com ressecção endoscópica reduz o tempo de internação hospitalar, dor no pós-operatório e complicações pulmonares associadas à cirurgia aberta. Entretanto, a abordagem cirúrgica é mais segura nos casos de lipomas gigantes e que apresentam vasos de grande calibre em seu pedículo. Entre as abordagens cirúrgicas, podemos lançar mão do acesso transoral, transtorácico e transcervical, sendo este último o realizado em nosso paciente, em virtude da familiaridade da equipe cirúrgica com esta via de acesso. Independentemente da técnica escolhida para a ressecção, por via endoscópica ou cirúrgica aberta, as taxas de recorrência do lipoma esofágico são baixas ${ }^{5}$.

\section{REFERÊNCIAS}

1. Qinying W, Wei L, Shuihong Z. Large pedunculated lipoma of the esophagus: Report of a case and review of literature. J Cancer Res Ther. 2015;11(4):1031. doi: 10.4103/09731482.154089 .

2. Feldman J, Tejerina M, Hallowell M. Esophageal lipoma: a rare tumor. J Radiol Case Rep. 2012;6(7):17-22. doi:10.3941/jrcr.v6i7.1015.

3. Cuttitta A, Annese V, Tancredi A, Clemente C, Scaramuzzi R. Giant esophageal lipoma. Updates Surg. 2011;63(2):125-7. doi:10.1007/s 13304-011-0044-0.

4. Jo DH, Chon HK, Woo SH, Kim TH. Endoscopic Resection of a Giant Esophageal Lipoma Causing Sudden Choking. Korean J Gastroenterol. 2016;68(4):210-3. doi:10.4166/kjg.2016.68.4.210.

5. Liu C-H, Chang H-C, Goan Y-G. Large pedunculated lipoma of the esophagus. J Formos Med Assoc. 2008;107(5):4247. doi: 10.1016/S0929-6646(08)60109-6.

Fonte de financiamento: Não

Conflito de interesses: Não

Data de Submissão: 31 Julho 2020

Decisão final: 26 Setembro 2021

\section{Autor de Correspondência:}

Bruno Amantini Messias

E-mail: bruno22med@hotmail.com 